Supplement of Biogeosciences, 14, 4409-4422, 2017

https://doi.org/10.5194/bg-14-4409-2017-supplement

(C) Author(s) 2017. This work is distributed under

the Creative Commons Attribution 3.0 License.

(c) (1)

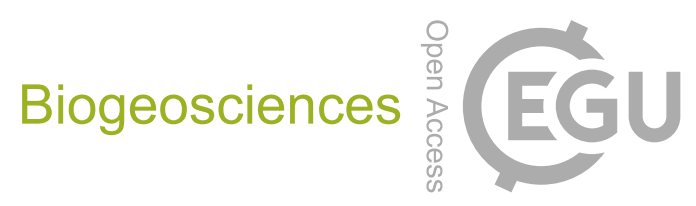

Supplement of

\title{
Response of water use efficiency to summer drought in a boreal Scots pine forest in Finland
}

Yao Gao et al.

Correspondence to: Yao Gao (yao.gao@fmi.fi)

The copyright of individual parts of the supplement might differ from the CC BY 3.0 License. 

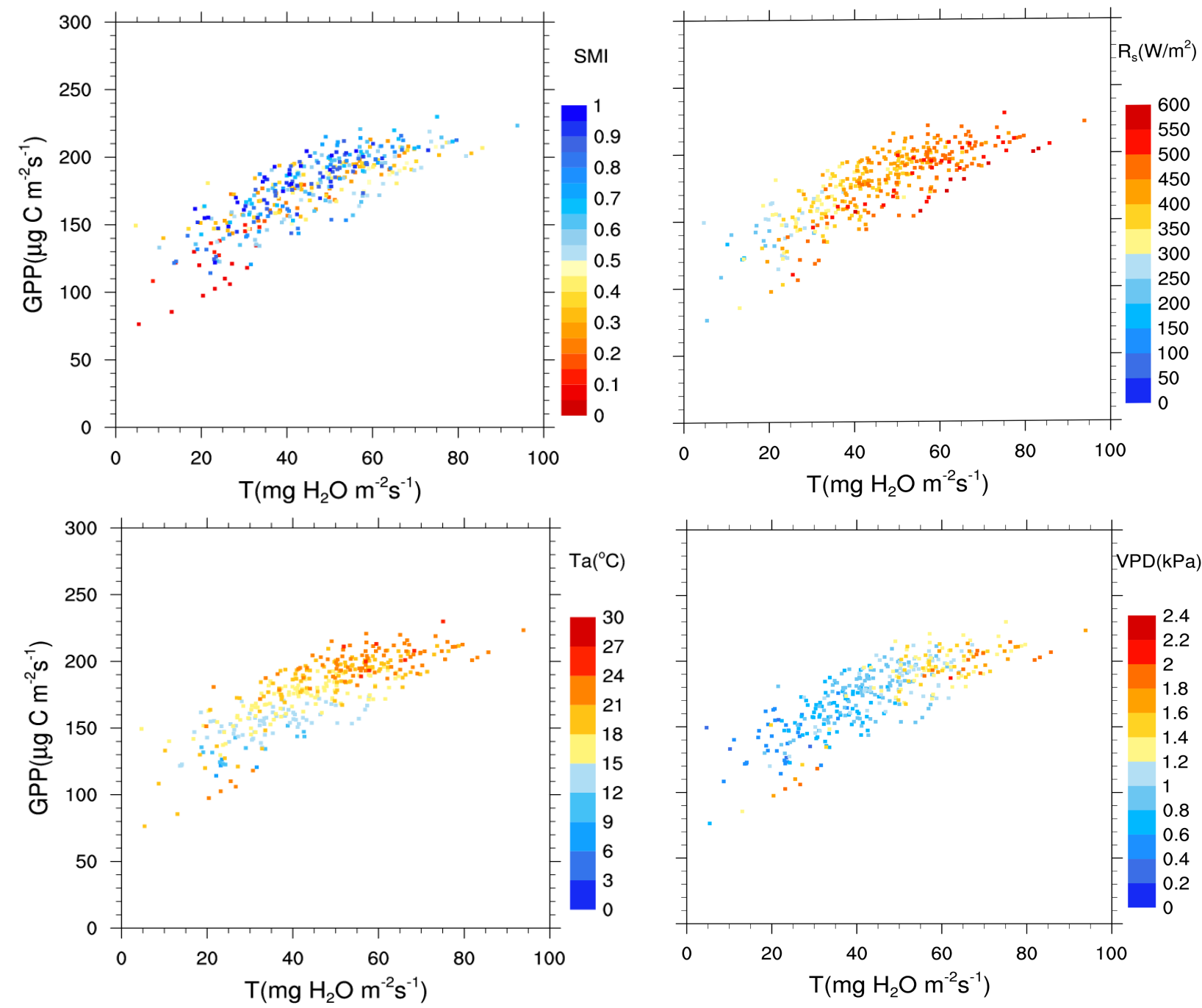

10 Figure S1: Relationship between the daytime averaged gross primary production (GPP in $\left.\mu \mathrm{g} \mathrm{C} \mathrm{m}^{-2} \mathrm{~s}^{-1}\right)$ and transpiration $\left(\mathrm{T}_{\text {in }} \mathrm{mg} \mathrm{H}_{2} \mathrm{O} \mathrm{m}^{-2} \mathrm{~s}^{-1}\right)$ at $\mathrm{Hyytiälä}$ in the summer months (June, July, August) of the 11-year study period (from 1999 to 2009) from the JSBACH simulation. Data are categorized according to daily mean soil moisture index (SMI), daytime mean incoming shortwave radiation $\left(\mathbf{R}_{\mathrm{s}}\right)$, daytime mean air temperature $\left(\mathbf{T}_{\mathrm{a}}\right)$ and daytime mean vapour pressure deficit (VPD), respectively. 
(a) Observation

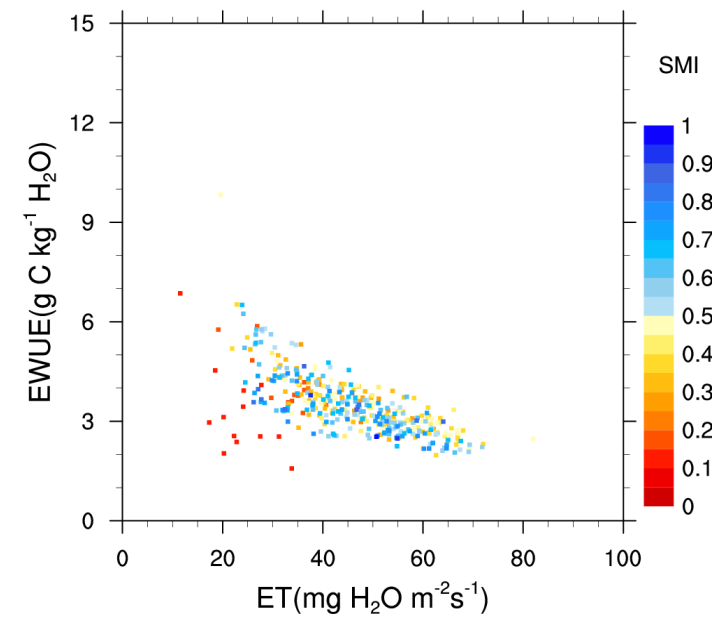

(b) Simulation

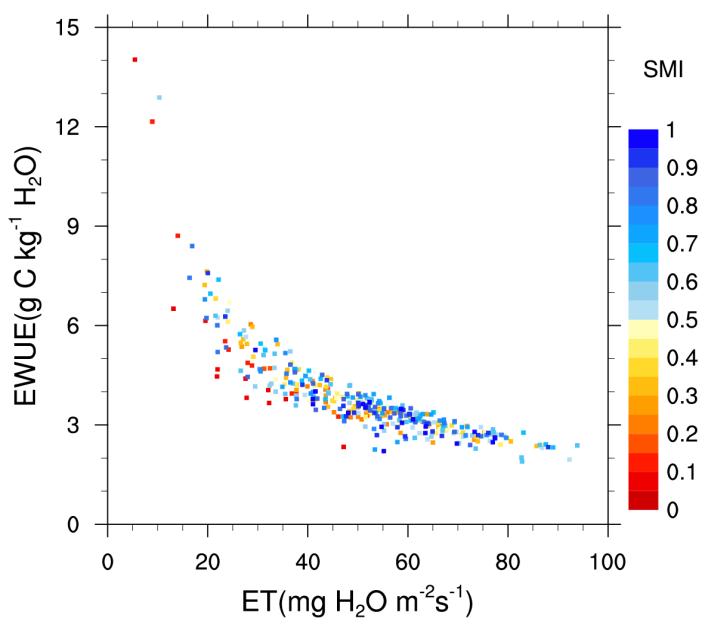

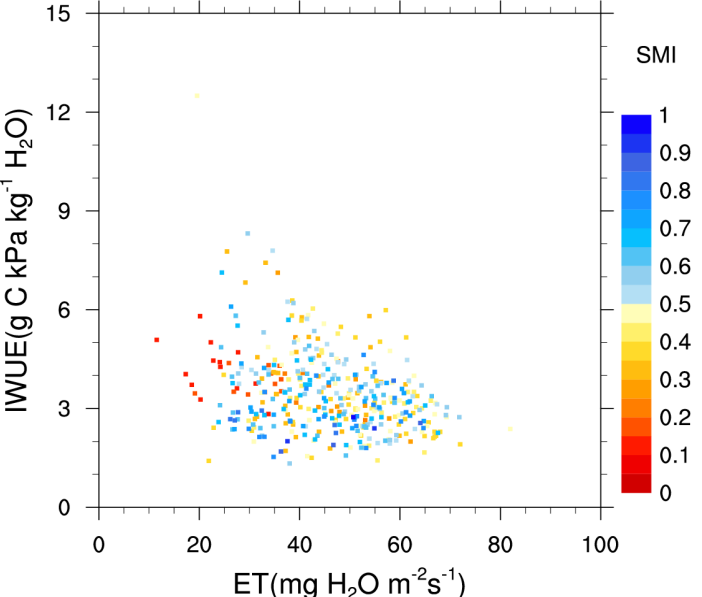
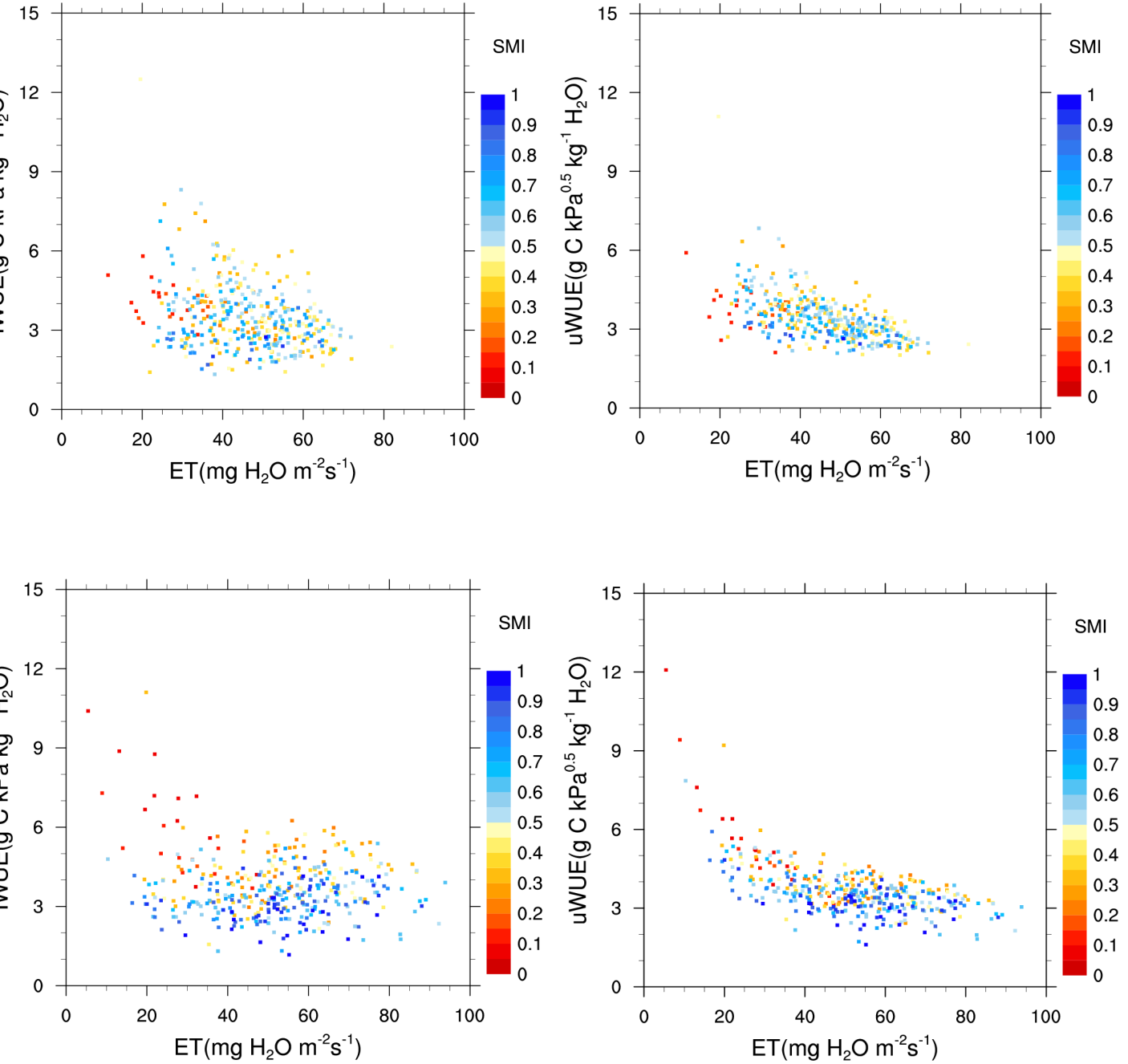

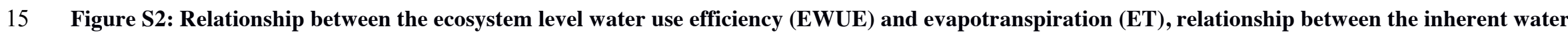
use efficiency (IWUE) and ET, and relationship between the underlying water use efficiency (uWUE) and ET at Hyytiälä in the summer months (June, July, August) of the 11-year study period (from 1999 to 2009) from (a) observation and (b) the JSBACH simulation. 

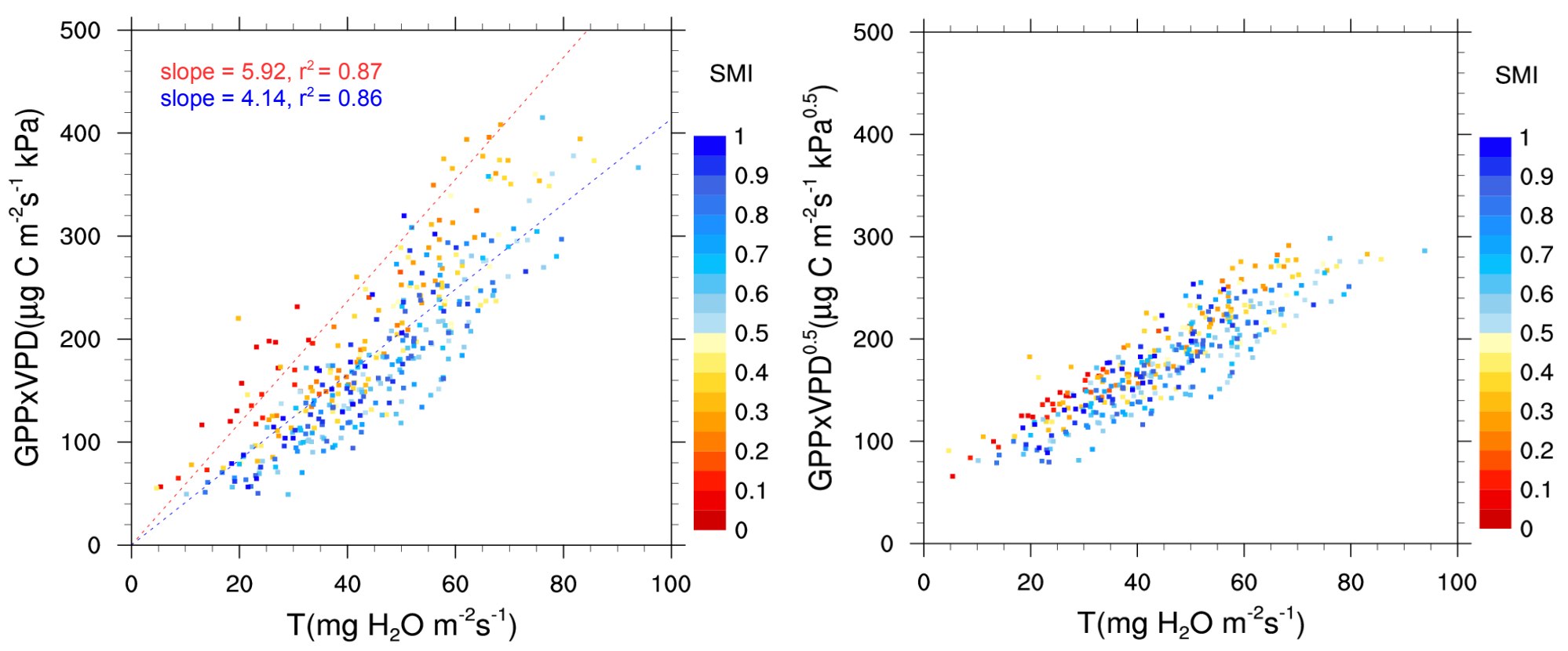

Figure S3: The dependence of the product of daytime mean gross primary production (GPP in $\mu \mathrm{g} \mathrm{C} \mathrm{m}^{-2} \mathrm{~s}^{-1}$ ) and daytime mean vapour pressure deficit

(VPD) on transpiration ( $\mathrm{T}$ in $\mathrm{mg} \mathrm{H} \mathrm{H}_{2} \mathrm{~m}^{-2} \mathrm{~s}^{-1}$ ) (i.e., GPP $\times \mathrm{VPD} / \mathrm{T}$, which represents the transpiration-based inherent water use efficiency), and the dependence of the production of GPP and the square root of VPD on T (i.e., GPP VPD ${ }^{0.5} / E T$, which represents the transpiration-based underlying water use efficiency) in the summer months (June, July, August) of the 11-year study period (from 1999 to 2009) from the JSBACH simulation. Data are categorized according to daily mean soil moisture index (SMI). The fitted lines for the dependence of the product of GPP and VPD on T are for the data under SMI $<0.2$ (red line) and data under $0.2 \leq$ SMI $<1$ (blue line); the fittings are statistically significant (p-value $<0.01$ ). No lines were fitted for the dependence of the production of GPP and the square root of VPD on ET, as the data under SMI $<0.2$ and data under $0.2 \leq$ SMI $<1$ are more converged in a line in comparison to the dependence of the product of GPP and VPD on T. 

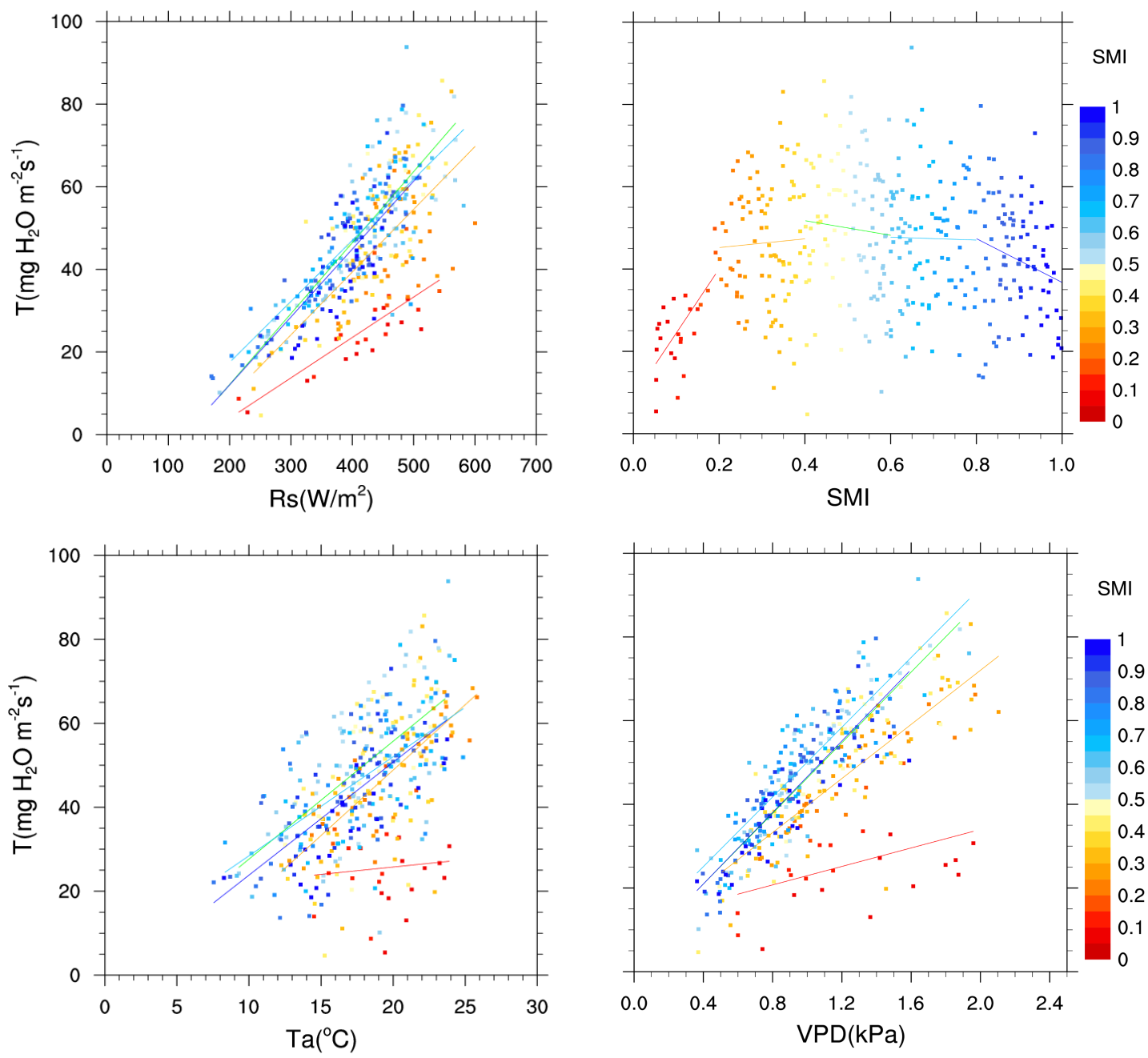

Figure S4: Response of daytime mean transpiration ( $T$ in $\left.\mathrm{mg} \mathrm{H}_{2} \mathrm{O} \mathrm{m}^{-2} \mathrm{~s}^{-1}\right)$ to daytime mean incoming shortwave radiation $\left(\mathrm{R}_{\mathrm{s}}\right.$ ), daytime mean air temperature $\left(\mathrm{T}_{\mathrm{a}}\right)$, daytime mean vapour pressure deficit (VPD), and daily mean soil moisture index (SMI) at Hyytiälä, categorized by daily mean soil moisture index (SMI) in the summer months (June, July, August) of the 11-year study period (from 1999 to 2009) from the JSBACH simulation. The regression lines are fitted for the five SMI groups (very dry: $0 \leq$ SMI $<0.2$, moderate dry: $0.2 \leq$ SMI $<0.4$, mid-range: $0.4 \leq$ SMI $<0.6$, moderate wet: 0.6 $\leq$ SMI $<0.8$, very wet: $0.8 \leq$ SMI $<1)$. 
Table S1: Regression parameters, correlation coefficient and statistical significance (p-value) of the dependences of the daytime averaged gross primary production (GPP in $\mu \mathrm{g} \mathrm{C} \mathrm{m}^{-2} \mathrm{~s}^{-1}$ ), evapotranspiration (ET in $\mathrm{mg} \mathrm{H}_{2} \mathrm{O} \mathrm{m}^{-2} \mathrm{~s}^{-1}$ ), transpiration ( $\mathrm{T}$ in $\mathrm{mg} \mathrm{H}_{2} \mathrm{O} \mathrm{m}^{-2} \mathrm{~s}^{-1}$ ) on the environmental variables (daytime mean incoming shortwave radiation $\left(R_{s}\right)$, daytime mean air temperature $\left(T_{a}\right)$, daytime mean vapour pressure deficit (VPD) and daily mean soil moisture index (SMI)) for the five SMI groups (N1: very dry $(0 \leq \mathrm{SMI}<0.2)$, N2: moderate dry $(0.2 \leq \mathrm{SMI}<0.4)$, N3: mid-range $(0.4 \leq$ SMI < 0.6$)$, N4: moderate wet $(0.6 \leq \mathrm{SMI}<0.8)$, N5: very wet $(0.8 \leq \mathrm{SMI}<1))$. The regressions with p-values smaller than 0.05 are considered to be statistically significant.

\begin{tabular}{|c|c|c|c|c|c|c|c|c|c|c|c|c|c|c|c|c|c|c|}
\hline \multirow{2}{*}{\multicolumn{3}{|c|}{$\begin{array}{c}\text { Environmental variable } \\
\text { Fitting function }\end{array}$}} & \multicolumn{4}{|c|}{$\mathrm{R}_{\mathrm{s}}$} & \multicolumn{4}{|c|}{$\mathrm{T}_{\mathrm{a}}$} & \multicolumn{4}{|c|}{ SMI } & \multicolumn{4}{|c|}{ VPD } \\
\hline & & & \multicolumn{4}{|c|}{ Linear function } & \multicolumn{4}{|c|}{ Linear function } & \multicolumn{4}{|c|}{ Linear function } & \multicolumn{4}{|c|}{ Linear function } \\
\hline & Parameter & & slope & interception & $\mathrm{r}$ & $\begin{array}{c}\mathrm{p}- \\
\text { value }\end{array}$ & slope & interception & $\mathrm{r}$ & $\begin{array}{c}\mathrm{p}- \\
\text { value }\end{array}$ & slope & interception & $\mathrm{r}$ & $\begin{array}{c}\mathrm{p}- \\
\text { value }\end{array}$ & slope & interception & $\mathrm{r}$ & $\begin{array}{c}\mathrm{p}- \\
\text { value }\end{array}$ \\
\hline \multirow{10}{*}{ GPP } & \multirow{5}{*}{ observation } & N1 & -0.01 & 102.90 & 0.03 & 0.87 & -12.01 & 366.27 & 0.80 & 0.00 & 1460.45 & -124.09 & 0.79 & 0.00 & -64.20 & 174.77 & 0.74 & 0.00 \\
\hline & & $\mathrm{N} 2$ & 0.06 & 133.75 & 0.18 & 0.08 & -0.88 & 174.34 & 0.14 & 0.21 & -42.30 & 172.26 & 0.08 & 0.44 & -12.19 & 170.66 & 0.20 & 0.04 \\
\hline & & $\mathrm{N} 3$ & -0.02 & 164.47 & 0.05 & 0.48 & -0.54 & 168.45 & 0.08 & 0.35 & -78.55 & 197.20 & 0.21 & 0.01 & -22.38 & 182.51 & 0.39 & 0.00 \\
\hline & & N4 & -0.05 & 168.20 & 0.19 & 0.05 & 0.63 & 135.69 & 0.11 & 0.23 & -69.42 & 194.10 & 0.17 & 0.07 & -17.90 & 163.61 & 0.24 & 0.01 \\
\hline & & N5 & -0.32 & 265.49 & 0.62 & 0.02 & 3.51 & 88.58 & 0.39 & 0.18 & 20.96 & 123.46 & 0.03 & 0.91 & -0.635 & 142.06 & 0.00 & 0.99 \\
\hline & \multirow{5}{*}{ simulation } & N1 & 0.18 & 52.37 & 0.57 & 0.00 & -3.40 & 193.31 & 0.35 & 0.09 & 530.44 & 70.53 & 0.92 & 0.00 & -6.21 & 135.05 & 0.10 & 0.64 \\
\hline & & $\mathrm{N} 2$ & 0.14 & 118.37 & 0.48 & 0.00 & 4.67 & 89.85 & 0.85 & 0.00 & 38.86 & 167.46 & 0.11 & 0.31 & 34.72 & 137.58 & 0.74 & 0.00 \\
\hline & & $\mathrm{N} 3$ & 0.17 & 107.09 & 0.65 & 0.00 & 5.49 & 79.98 & 0.85 & 0.00 & -50.86 & 204.00 & 0.15 & 0.17 & 47.16 & 127.10 & 0.77 & 0.00 \\
\hline & & N4 & 0.18 & 109.55 & 0.56 & 0.00 & 5.90 & 76.51 & 0.91 & 0.00 & -5.48 & 186.77 & 0.00 & 0.90 & 67.48 & 119.83 & 0.79 & 0.00 \\
\hline & & N5 & 0.25 & 79.70 & 0.72 & 0.00 & 6.06 & 73.98 & 0.91 & 0.00 & 41.25 & 139.15 & 0.09 & 0.40 & 70.47 & 112.84 & 0.84 & 0.00 \\
\hline \multirow{10}{*}{ ET } & \multirow{5}{*}{ observation } & N1 & 0.05 & 8.71 & 0.59 & 0.00 & -0.96 & 46.15 & 0.31 & 0.13 & 266.19 & -13.41 & 0.71 & 0.00 & -0.93 & 28.12 & 0.00 & 0.81 \\
\hline & & $\mathrm{N} 2$ & 0.05 & 27.58 & 0.29 & 0.00 & 0.69 & 34.18 & 0.20 & 0.05 & 44.63 & 31.87 & 0.17 & 0.10 & 3.05 & 43.68 & 0.01 & 0.31 \\
\hline & & N3 & 0.03 & 35.14 & 0.24 & 0.00 & 0.87 & 32.94 & 0.22 & 0.01 & -18.35 & 58.58 & 0.09 & 0.27 & 3.42 & 45.73 & 0.01 & 0.19 \\
\hline & & N4 & 0.05 & 22.96 & 0.36 & 0.00 & 1.35 & 21.74 & 0.48 & 0.00 & 32.45 & 21.96 & 0.16 & 0.09 & 12.98 & 31.72 & 0.36 & 0.00 \\
\hline & & N5 & -0.06 & 66.55 & 0.30 & 0.31 & 2.33 & 7.57 & 0.66 & 0.01 & 71.61 & -19.03 & 0.30 & 0.31 & 25.31 & 23.20 & 0.49 & 0.09 \\
\hline & \multirow{5}{*}{ simulation } & N1 & 0.12 & -20.16 & 0.79 & 0.00 & 0.50 & 19.13 & 0.11 & 0.61 & 148.14 & 12.95 & 0.55 & 0.01 & 12.69 & 13.15 & 0.42 & 0.04 \\
\hline & & $\mathrm{N} 2$ & 0.17 & -22.60 & 0.73 & 0.00 & 2.63 & 1.03 & 0.60 & 0.00 & 9.30 & 48.59 & 0.03 & 0.76 & 29.51 & 15.87 & 0.79 & 0.00 \\
\hline & & $\mathrm{N} 3$ & 0.18 & -23.07 & 0.88 & 0.00 & 2.41 & 11.19 & 0.47 & 0.00 & -22.97 & 66.03 & 0.08 & 0.44 & 38.30 & 12.85 & 0.79 & 0.00 \\
\hline & & N4 & 0.19 & -22.89 & 0.86 & 0.00 & 2.10 & 15.07 & 0.48 & 0.00 & -24.13 & 69.57 & 0.08 & 0.41 & 45.11 & 10.77 & 0.78 & 0.00 \\
\hline & & N5 & 0.19 & -18.58 & 0.84 & 0.00 & 2.52 & 10.04 & 0.59 & 0.00 & 60.27 & -1.50 & 0.20 & 0.05 & 43.32 & 13.64 & 0.81 & 0.00 \\
\hline \multirow{5}{*}{$\mathrm{T}$} & \multirow{5}{*}{ simulation } & N1 & 0.10 & -15.60 & 0.78 & 0.00 & 0.35 & 18.73 & 0.09 & 0.68 & 156.34 & 8.79 & 0.67 & 0.00 & 11.04 & 11.90 & 0.44 & 0.03 \\
\hline & & $\mathrm{N} 2$ & 0.15 & -21.42 & 0.68 & 0.00 & 3.11 & -13.46 & 0.72 & 0.00 & 10.59 & 43.10 & 0.03 & 0.73 & 32.32 & 7.39 & 0.88 & 0.00 \\
\hline & & N3 & 0.17 & -22.26 & 0.84 & 0.00 & 2.81 & -0.41 & 0.56 & 0.00 & -17.76 & 58.86 & 0.06 & 0.54 & 42.22 & 4.06 & 0.89 & 0.00 \\
\hline & & N4 & 0.15 & -12.08 & 0.79 & 0.00 & 2.36 & 4.86 & 0.63 & 0.00 & -3.58 & 49.90 & 0.01 & 0.88 & 41.66 & 8.44 & 0.84 & 0.00 \\
\hline & & N5 & 0.16 & -20.81 & 0.82 & 0.00 & 2.69 & -3.05 & 0.70 & 0.00 & -53.28 & 90.02 & 0.20 & 0.06 & 42.72 & 3.87 & 0.88 & 0.00 \\
\hline
\end{tabular}

